\title{
Practices of tracheal suctioning technique among health care professionals: Literature review
}

\author{
Rozina Khimani \\ Aga Khan University, rozina.khimani@aku.edu \\ Fauziya Ali Dr. \\ Aga Khan University, fauziya.ali@aku.edu \\ Salma Rattani \\ Aga Khan University, salma.rattani@aku.edu \\ Mohammad Sohail Awan \\ Aga Khan University, sohail.awan@aku.edu
}

Follow this and additional works at: https://ecommons.aku.edu/pakistan_fhs_mc_surg_surg

Part of the Nursing Commons, Otolaryngology Commons, and the Surgery Commons

\section{Recommended Citation}

Khimani, R., Ali, F., Rattani, S., Awan, M. (2015). Practices of tracheal suctioning technique among health care professionals: Literature review. International Journal of Nursing Education, 7(1), 179-183.

Available at: https://ecommons.aku.edu/pakistan_fhs_mc_surg_surg/858 


\title{
Practices of Tracheal Suctioning Technique among Health Care Professionals: Literature Review
}

\author{
Rozina Khimani ${ }^{1}$, Fauziya Ali $^{2}$, Salma Rattani ${ }^{3}$, Sohai Awan ${ }^{4}$ \\ ${ }^{1}$ Clinical Nurse Instructor, Aga Khan University Hospital, ${ }^{2}$ Assistant Professor, ${ }^{3}$ Assistant Professor and Director, \\ Aga Khan University School of Nursing and Midwifery, ${ }^{4}$ Associate Professor and Section Head, Otolaryngology \\ Department of Surgery, Aga Khan University, Karachi Pakistan
}

\begin{abstract}
The current study aims to assess tracheal suctioning practices among health care practitioners; nurses, critical care technicians and physiotherapist. Employing literature review as the methodology, multiple databases were searched focusing on three phases of tracheal suctioning (a) the pre suctioning phase, (b) the suctioning phase, (c) the post suctioning phase and complications related to tracheal suctioning. It was concluded that to provide quality care it is important that the evidence based practice guidelines should be followed.
\end{abstract}

Keywords: Tracheal Suctioning, Tracheostomy, Evidence Based Practice, Nursing Skills, Health Care Professionals

\section{INTRODUCTION}

Tracheal suctioning is one of the critical nursing interventions to keep the airway patent by removing secretion via suctioning. This critical skill requires expertise with the knowledge to perform and facilitate a patient's effortless breathing pattern through effective secretion management. But this aspect of care is associated with the risk of injury therefore, the role of competent health care professionals (HCP) is vital in performing tracheal suctioning.

The literature reviewed was obtained through different databases that includes: Cumulative Index to Nursing and Allied Health Literature (CINAHL), Medical Literature Analysis and Retrieval System Online (MEDLINE), PubMed, Science Direct, SpringerLink, and Google Scholar.

\section{Pre suctioning phase}

Prior to suctioning, patient assessment; monitoring the vital signs and chest auscultation is important ${ }^{1,2,3}$. The reommended period for assessment is in each shift, before each suctioning, or depending on the patient's

\section{Corresponding author:}

\section{Rozina Khimani}

Aga Khan University Hospital, School of Nursing and Midwifery, Stadium Road, Karachi 74800, Pakistan

Phone: +92 2134930051

Email: rozina.khimani@aku.edu need 1,2,4. Through evidence-based practices it is revealed that nurse are using their clinical judgment, nurses perform tracheal suctioning without using normal saline and they screen patients' cardiopulmonary position before, during, and after the suctioning phase ${ }^{5}$. Similarly an observational study conducted to assess the nurses' practices of tracheal suctioning in the cardiac intensive care unit (CICU) and the general intensive care unit (GICU) in Ireland, it was revealed that among the CICU nurses $(n=17)$ only two nurses, i.e. $12 \%$ out of 34 , from CICU and four nurses, i.e. $14 \%$, from GICU performed chest auscultation to assess the need of suctioning ${ }^{7}$.

While looking the entire pool as health care practitioners it was revealed that the patients with respiratory distress were monitored for occurance of tachypnea and deoxygenation throughout the suctioning process and based on patient's need, the HCPs selected a suitable sized of suctioning catheter ${ }^{6}$.

Along with assessment, informing the patient and taking consent prior to the suctioning have been identified as strategies for reducing the anxiety and distress of patients as they help in gaining maximum outcomes from suctioning ${ }^{1,2}$. It has been found that informing a client about the details of the procedure always ensures its smooth execution. Whereas, one study explicated that, out of $53,28 \%$ of the nurses from the GICU were unable to communicate and explain procedures to the patients ${ }^{7}$. 
Hypoxemia is one of the complications related to tracheal suctioning ${ }^{2,8}$. Hypoxemia is associated with cardiac arrhythmias, hypotension, cardiac arrest, and death, therefore, hyperventilation and hyperoxygenation are essential aspects of treatment ${ }^{1}$.

Hyperoxygenation is the administration of oxygen in greater amount than what the patient is receiving or requires ${ }^{9}$. Hyperoxygenation and hyperinflation is found to be an effective intervention prior to suctioning in order to prevent suction induced hypoxia in adult patients ${ }^{10}$. However, the careful oxygen administration is required in patients with chronic obstructive pulmonary diseases (COPD), as these patients cannot tolerate high oxygen flow ${ }^{11}$. Tracheal suctioning and tracheostomy care are high-risk processes. To avoid adverse consequences, HCPs who execute these, whether they are experts or beginners, must follow the evidence based guidelines. It is also suggested that, patients should be hyperoxygenated and encouraged deep breathing, then manage four to six compressions with a manual ventilator bag or trigger the ventilator for hyperoxygenation ${ }^{4}$.

Another point to note during this phase is that, normal saline instillation is not recommended prior to or during suction. It is usually a misconception that it helps in liquifying secretions $9,4,8$. The potential hazards of normal saline instillation include a fall in $\mathrm{PaO} 2$, lower respiratory track infection, and failure to remove all saline during suctioning ${ }^{9,12}$. Despite available evidences against the use of saline in tracheal suctioning, a survey found that $33 \%$ of nurses and therapists still use saline before and during suctioning ${ }^{4}$. In fact, another survey, on 1665 nurses and respiratory therapists from 27 different sites in the United States, regarding saline instillation protocol, reported that $74 \%$ centers had a protocol which recommended saline instillation during suctioning ${ }^{13}$.

A quasi experimental study was conducted to assess the level of dyspnea with the use of saline during suctioning in which dyspnea was graded immediately after the suctioning, and at 10,20, and 30-minute intervals. The findings indicated no beneficial use of saline; whereas, age variation highlighted that older patients, above 60years of age had decreased levels of dyspnea when suctioning was done without saline instillation. The study validated that saline instillation might precipitate a considerable increase in the level of dyspnea for up to 10 minutes after suctioning in patients older than 60 years of age ${ }^{14}$. Similarly, the findings of another study reported that the use of saline instillation during tracheal suctioning decreased the mix venous saturation as compared to the patients, suctioning without saline instillation ${ }^{5}$.

The use of saline during suctioning also varies among health care professionals, such as nurses, doctors, and physiotherapists. Survey on saline instillation protocol and related practices of HCPs indicated that $79 \%$ hospitals use saline during suctioning, among which $58 \%$ physiotherapists use saline in their practices, while saline use by nurses and other medical staff was $42 \%{ }^{15}$.

Pain is also one of the frequently associated complaint during tracheal suctioning ${ }^{16}$. Tracheal suctioning was specifically an identified discomforting factor among $30 \%$ of the ICU ventilated patients. On a 0-10 scale, pain intensity during suctioning among patients with tracheostomy was reported to be at 7 or greater ${ }^{17}$.

Tracheal suctioning is an invasive and sterile procedure, therefore, all aseptic protocols, such as, hand washing before and after the procedure, gloves, apron, protectors and use of sterile catheter for each episode of suction should be followed to prevent patients from getting an infection ${ }^{2,4}$. Several diseases can occur during suctioning treatment due to noncompliance of standard precautions. The sterile procedure is essentially followed to avoid contamination of the airway. The tube must never be reused once it is opened and a new sterile catheter must be used for each session of suctioning ${ }^{18}$.

Compliance of infection control practices among HCPs needs to be emphasized to prevent complications related to the suctioning procedure and equipment. A study conducted in Ireland identified that, out of 53 , only $30 \%$ of the nurses in GICU and $65 \%$ nurses, from 34 nurses, in CICU performed handing washing prior to suctioning. Moreover, 59\% CICU and $29 \%$ of GICU nurses failed to maintain the sterility of the suction catheter till its insertion into the airway, which indicates an alarming situation ${ }^{7}$.

Another study was conducted on Infection Prevention (Werkgroep Infectiepreventie [WIP]) to find out whether some policies on tracheal suctioning (open and closed tracheal suctioning systems) are superior to others, in terms of anticipation of ventilatorassociated pneumonia (VAP). They found that there was no significant difference in the use of both open and closed tracheal suction systems in decreasing the rate of VAP. The study, however, clarifies that the 
quality of the evidence is substantive. Investigations other than the anticipation of VAP should regulate the choice of the suction system ${ }^{6,19}$.

\section{The suctioning phase}

The suctioning phase is an active period for the removal of secretions. It is widely accepted that during tracheal suctioning, the catheter diameter should be one and a half times of the tracheotomy tube in diameter ${ }^{1,4}$. The tracheostomy suction catheter size can be easily calculated. The size of tracheotomy tube divided by 2 and multiplied by 3 will give the appropriate catheter size according to the tracheostomy tube $((14,4)$. However, a study in two critical care areas in Ireland documented that $40 \%$ of the CICU nurses and $28 \%$ of the GICU nurses selected a larger sized suction catheter in comparison to the size of tracheotomy tube ${ }^{7}$. Large catheter obstructs the airway resulting in hypoxemia during suctioning as well as trauma to the mucosal lining of the trachea ${ }^{1,2}$.

Negative pressure is needed to remove tracheal secretions. Most of the literature recommends that the pressure range during tracheal suctioning should be from 70 to $150 \mathrm{mmHg}^{1,9,2}$. However, it has been suggested that a pressure range between 70 to $120 \mathrm{mmHg}^{20,4}$. Furthermore, a pressure of $200 \mathrm{~mm} \mathrm{Hg}$ can cause mucosal lining damage, alveolar collapse, and could also lead to bacterial colonization ${ }^{2,9}$.

The whole procedure of tracheal suctioning should not take too long as it could cause hypoxia, bradycardia, and mucosal damage 11,1,9,2. The recommended duration of tracheal suctioning is 10 to 15 seconds with 20 to 30 seconds intervals between passes ${ }^{1,4,8}$. Each session of suctioning should comprise of three suction catheter passes at the most ${ }^{11,1,9,2}$.

Furthermore, suction vacuum should always be applied during catheter withdrawal ${ }^{2}$. Moreover, the suction catheter should not be inserted deeply into the trachea as it causes cough and vagal nerve stimulation at the bifurcation of the trachea (Carina). Where catheter encounter resistance, withdraw the catheter $1 \mathrm{~cm}$ and then applies suction ${ }^{1,2}$.

Tracheal suctioning can easily cause airway mucosal trauma when inappropriate suctioning methods are used. Suction can only be useful for extracting secretion from the airway. Unnecessary high vacuum pressure and extensive suction exercises should be avoided. It is important to pay special consideration to the possibility of complexity in patients who might be predominantly susceptible to mucosal injury, i.e., very young patients, or patients with thrombocytopenia or on general anticoagulant therapy ${ }^{18}$.

A randomized control trial study was conducted to relate and distinguish the alterations in endotracheal suction consequences in patients who routinely received 2 hourly suctioning and those who received it following an evaluation. The results verified a clear rise in nurses' knowledge regarding endotracheal suctioning and indicated the effectiveness of procedures with minimal complications ${ }^{3}$.

Suctioning is a high risk process, which can cause hypoxemia, bleeding, cardiovascular instability, infection, atelectasis, elevated intracranial pressure, and can also create lesions in the tracheal mucosa. These complications can be avoided with the use of best practices ${ }^{21}$.

\section{Post suctioning phase}

The patient's condition needs to be assessed following suctioning to evaluate the effectiveness of the procedure, which includes respiratory rate, oxygen saturation, and chest auscultation for the presence of secretions and bilateral air entry to the lungs $1,2,4,8,9$. Oxygen therapy needs to be reconnected immediately after suctioning, ideally, within 10 seconds to prevent the patient from hypoxia ${ }^{1,2}$.

Moreover, post suctioning documentation is essential which includes patient physiological and psychological response, color, odor, consistency and amount (COCA) of secretion ${ }^{4,9}$.

\section{Complications related to tracheal suctioning}

Endotracheal suctioning is, thus, imperative in order to decrease the danger of atelectasis and consolidation that might lead to insufficient ventilation. A number of risk and complications are related to the suction process. These include atelectasis, bleeding, cardiovascular instability, infection, hypoxemia, elevated intracranial pressure, and grazes in the tracheal mucosa. The key recommendations use evidence based guidelines to prevent and minimize these complications ${ }^{21}$.

A conducted a study with the objective of determining the impact of suction tube insertion and tracheal stimulation on the cerebrovascular and systemic vascular status in adults with severe 
traumatic brain injury. They tested thirty intubated and mechanically ventilated adults with brain injury. The participants' average age was $31+/-15$ years. The results showed that suction tube inclusion and tracheal stimulation, inaccessible from other constituents of the suctioning process, meaningfully enlarged cerebral perfusion pressure (CPP), mean arterial pressure (MAP), and mean intracranial pressure (MICP) ${ }^{22}$.

Moreover, a tracheostomy team approach can minimize the risk of complications pertinent to tracheal suctioning for which a study examined the mechanisms of tracheostomy through an inter professional team approach which consist of divers health cre professional possess expertise to care patients with tracheostomy. The findings indicated that tracheotomy teams increased the regularity of care through the expansion and application of inter professional protocol. These findings provided new ways of understanding the role of tracheostomy teams in effectively implementing complex protocols and mechanisms through which inter professional teams might produce positive consequences for tracheotomy patients ${ }^{23}$.

\section{CONCLUSION}

Tracheal suctioning is an important aspect of airway management however, this aspect of care is associated with many rist factors therefore, its is important for health care professionals to follow evidence based guidelines to prevent complications and promote safety. Moever, the knowledge and competence of health care professionals play an important role in enhancing safety of the patient.

Acknowledgement: I (Rozina Khimani, principal investigator) would like to acknowledge Division of Nursing Services, Aga Khan University, Hospital, Karachi, Pakistan.

Conflict of Interest: The authors declare having no conflict of interest.

Source of Funding: No separate funding was received for this study as the current study was done during graduate studies.

\section{REFERENCES}

1. Day, T., Farnell, S., Haynes, S., Wainwright, S., \& Wilson-Barnett, J. (2002). Tracheal suctioning: an exploration of nurses' knowledgeand competence in acute and high dependency ward areas. Journal of Advanced Nursing, 39 (1), 35-45.

2. Freeman, S. (2011). Care of adult patients with a temporary trachesotomy. Nursing Standard,26 (2), 49-56.

3. Nance-Floyd, B. (2011). Tracheostomy care: An evidence-based guide to suctioning and dressing change. Journal of American Nurse Today , 6 (7), 1-3.

4. Wood, C. J. (1998). Endotracheal suctioning: a literature review. Journal of Intensive and Critical Care Nursing, 14, 124-136.

5. Kuriakose, S. A. (2008). Using the synergy model as best practice in endotracheal tube suctioning of critically ill patients. Journal of Dimensions of Critical Care Nursing, 27(1), 10-15.

6. Niel-Weise, B. S., Snoeren, R. L., \& Broek, P. J. (2007). Policies for Endotracheal Suctioning of Patients Receiving Mechanical Ventilation: A Systematic Review of Randomized Controlled Trials. Journal of Infection Control and Hospital Epidemiology, 28(5), 529-536.

7. Kelleher, S., \& Andrews, T. (2006). An observational study on the open-system endotracheal suctioning practices of critical care nurses. Journal of Clinical Nursing, 17, 360-369.

8. Russell, C. (2005). Providing the nurse with a guide totracheostomy care and mangement. British Journal of Nursing , 14 (8), 428-433.

9. Day, T., Wainwright, S. P., \& Wilson-Barnett, J. (2001). An evaluation of teaching Intervention to improve the practice of endotracheal suctioning in intensive care unit. Journal of Clinical Nursing, 10, 682-696.

10. Atta, J. M., \& Beck, S. L. (1992). Preventing hypoxemia and hemodynamic compromise related to endotracheal suctioning. American Journal of Critical Care, 1 (3), 62-79.

11. Barnett, M. (2005). Tracheostomy Management and care. Journan of Community Nursing, 19 (1), 4-8.

12. Halm, M. A., \& Krisko-Hagel, K. (2008). Instilling Normal Saline with suctioning: Benificial technique or potencially harmful sacred cow? American Journal of Critical Care, 17(5), 469-472.

13. Sole, M. L., Byers, J. F., Zhang, Y., Banta, C. M., \& Brummel, K. (2003). A Multisite Survey of Suctioning Techniques and Airway Management Practices. American Journal of Critical Care, 12(3), 220-230.

14. O'Neal, P. V., Grap, M. J., Thompson, C., \& Dudley, W. (2001). Level of dyspnoea experienced in mechanically ventilated adults 
with and without saline instillation prior to endotracheal suctioning. Journal of Intensive and Critical Care Nursing, 17, 356-363.

15. Reeve, J. C., Davies, N., Freeman, J., \& Donovan, B. (2007). The use of normal saline instillation in the intensive care unit by physiotherapists: a survey of practice in New Zealand. Journal of Physiotherapy, 35(3), 119-125.

16. Arroyo-Novoa, C. M., Figueroa-Ramos, M. I., Puntillo, K. A., Stanik-Hutt, J., Thompson, C.

L., White, C., et al. (1998). Pain related to tracheal suctioningin awake acutely and critically ill adult: A descriptive study. Journal of Intensive and Crtical CareNursing, 24, 20-27.

17. Stotts, N. A., Puntillo, K., Stanik-Hutt, J., Thompson, C. L., White, C., \& Wild, L. R. (2007). Does age make a difference in procedural pain perceptions and responses in hospitalized adults? Journal of Acute Pain, 9, 125-134.

18. Florentini, A. (1992). Potential hazards of tracheobronchial suctioning. Intensive and Critical Care Nursing, 8(4), 217-226.

19. Jansson, M., Ala-Kokko, T., Ylipalosaari, P., \& Kyngas, H. (2013). Evaluation of endotracheal- suctioning practices of critical-care nurses - An observational correlation study. Journal of Nursing Education and Practice, 3(7), 99-105.

20. Bond, et al. (2003). Best practice in the care of patients with tracheostomy. Nursing Times, 99 (30), 24-25.

21. Pedersen, C. M., Rosendahl-Nielsen, M., Hjermind, J., \& Egerod, I. (2009). Endotracheal suctioning of the adult intubated patient-What is the evidence? Journal of Intensive and Critical Care Nursing, 25, 21-30.

22. Brucia, J., \& Rudy, E. (1996). The effect of suction catheter insertion and tracheal stimulation in adults with severe brain injury. Journal of Heart and Lung, 25(4), 295-303.

23. Mitchell, R., Parker, V., \& Giles, M. (2013). An interprofessional team approach to tracheostomy care: A mixed-method investigation into the mechanisms explaining tracheostomy team effectiveness. International Journal of Nursing Studies, 50, 536-542. 